\title{
Energy-resolved Compton scatter estimation for micro-CT
}

\author{
A.M.T. Opie ${ }^{* a}$, A.P.H. Butler ${ }^{a, b}$, and P.J. Bones ${ }^{a}$ \\ ${ }^{a}$ Dept. of Electrical and Computer Engineering, University of Canterbury, Christchurch, NZ \\ ${ }^{b}$ Centre for Bioengineering, University of Otago, Christchurch, NZ
}

\begin{abstract}
$\mathrm{X}$-ray scatter can cause significant distortion in CT imaging, especially with the move to cone-beam geometries. Incoherent scatter (Compton scatter) is known to reduce the energy of scattered photons according to the angle of the scattering. The emergence of energy-resolved x-ray detectors offers an opportunity to produce and apply more accurate scatter estimates, leading to improved image quality.

We have developed a scatter estimation algorithm that accounts for the variation in scatter with incident radiation energy. Where existing methods generate estimates of scatter for the complete detected energy band, our new method produces separate estimates for each of the energy bands that are measured, allowing a more focused correction of scatter. Our method is intended to be used in an iterative compensation framework like that of Rührnschopf and Klingenbeck (2011); it calculates the scatter contribution to each energy bin used in a scan based on the current volume estimate.

Comparisons with Monte Carlo simulations indicate that this algorithm is effective at estimating the scatter level in separate energy bins. We found that the amount of scatter that loses enough energy to hop between energy bands is small enough to neglect, but that scatter intensity is dependent on the incident energy, so application of a spectrally-aware compensation technique is valuable.
\end{abstract}

Keywords: Compton, scatter, estimation, spectral, micro-CT, energy-resolved

\section{INTRODUCTION}

The detection of scattered x-rays in a CT scan causes cupping and streaking artefacts in the reconstructed image. Hardware and software techniques exist to reduce or compensate for scatter, however hardware techniques are not suitable for micro-CT, and most software techniques do not account for energy-dependent scatter behaviour. We have created a fast analytical scatter estimation algorithm for energy-resolved micro-CT.

\subsection{Scatter}

As an x-ray photon travels through a material, there are three possibilities: it can pass through unaffected, it can be absorbed by an atom within the material by the photoelectric effect, or it can be scattered by an electron within the material. ${ }^{1}$ Scatter can be divided into two types: Rayleigh (coherent) scatter, ${ }^{2}$ where the photon does not lose any energy and is usually deflected only by a small angle, and Compton (incoherent) scatter, ${ }^{3}$ where the photon deposits some of its energy onto the electron with which it interacts and the angle of deflection can be anywhere between zero and 180 degrees. The key difference between the two types of scatter is that Compton scattering causes a photon to lose some of its energy while Rayleigh scattering does not.

The model used for reconstructing images from CT data operates on the principle that any photon that interacts with the object under study is removed from the photon beam and thus does not reach the detector. However, scattered photons are not removed, only deflected, so they can still reach the detector. When scattered photons reach the detector they increase the measured intensity and thus reduce the apparent attenuation of the material between the x-ray source and the detector. These corrupted attenuation values cause artefacts in the reconstructed CT image. ${ }^{4}$ The artefacts resulting from scatter are similar to those produced by beam hardening, ${ }^{5}$ namely streaks between high-density features and cupping.

Many methods have been proposed for reducing the effect of scatter. Hardware methods such as antiscatter grids $^{6}$ and air gaps ${ }^{7}$ are simple and can be effective, but they increase patient dose and space requirements,

*Corresponding author: alex.opie@pg.canterbury.ac.nz

Developments in X-Ray Tomography VIII, edited by Stuart R. Stock,

Proc. of SPIE Vol. 8506, 850616 - (C) 2012 SPIE - CCC code: 0277-786/12/\$18

doi: $10.1117 / 12.930319$

Proc. of SPIE Vol. $8506850616-1$ 
respectively. In addition, the detector pixel pitch used in micro-CT scanners is generally of the order of $50 \mu \mathrm{m}$ so an antiscatter grid would be unsuitable as the blades would obscure a significant proportion of each pixel. ${ }^{8}$

Beam-stop arrays can be used to directly measure scatter intensities within x-ray projections. ${ }^{9}$ Interpolating these measurements then produces an estimate of the scatter intensity in each image, which can be subtracted from the projection proper. While this method is effective in compensating for scatter, it requires at least a doubling of radiation exposure for the patient.

Various methods exist for estimating scatter contributions by operating directly on normal measured CT data. ${ }^{4,10-12}$ These 'projection-based' correction methods operate with varying degrees of success, but are generally not as accurate as 'reconstruction-based' correction methods. ${ }^{13}$ Reconstruction-based methods operate iteratively by reconstructing a volume, calculating a scatter estimate based on that volume, correcting for the scatter, then repeating. ${ }^{13}$ They tend to be Monte Carlo driven methods, which can be computationally expensive. Here we describe a reconstruction-based method with a smaller computational expense.

\subsection{Energy-resolved CT}

Traditional x-ray detectors measure the integral of the incident radiation spectrum. ${ }^{14}$ However, this approach neglects the information available in the variation of attenuation with energy. ${ }^{15} \mathrm{New}$ technologies are appearing that count individual photons and measure their respective energies, for example the Medipix family of detectors. ${ }^{16}$ These detectors provide photon counts in each of a finite number of 'energy bins'. They are being used in experimental CT scanners ${ }^{17}$ and research is active in assessing the advantages of spectral CT such as improved signal-to-noise ratio, ${ }^{18}$ reduced beam-hardening artefacts, ${ }^{19}$ material decomposition, ${ }^{20}$ and the concurrent use of multiple contrast agents. ${ }^{21}$

All of the post-acquisition scatter compensation techniques referred to in the previous subsection are designed to operate on data produced by traditional detectors. However, like primary radiation, scatter also varies with energy, and considering this should result in improved scatter compensation. The fact that Compton scatter reduces the energy of the radiation also suggests that considering the energy of scatter should be valuable.

We have developed an algorithm that estimates the scatter contribution to each energy bin based on a reconstructed volume. Our intention is for the algorithm to be used in an iterative framework like that described by Rürnschopf and Klingenbeck. ${ }^{13}$ The aim of this algorithm is to offer the accuracy of reconstruction-based estimation methods whilst being faster than a Monte Carlo simulation. We have chosen micro-CT as the target of our algorithm for two primary reasons. First, current energy-resolved detectors are mainly deployed in experimental micro-CT scanners, including the MARS scanner ${ }^{22}$ developed by our group. Second, micro-CT is not amenable to the use of antiscatter grids or large air gaps.

Section 2 contains a derivation of the mathematical model used by our new algorithm and some comments on its implementation. Section 3 shows the results of validation against a Monte Carlo system, then in section 4 we explain these results and discuss their implications. In section 5 we offer concluding thoughts and an outline of required future work related to the algorithm.

\section{ALGORITHM}

Our new algorithm performs an analytical calculation of the scatter intensity that is expected at the detector based on an estimate of the imaged volume. This algorithm differs from existing analytical methods in that it can produce an estimate for each of the energy bins being measured by an energy resolving x-ray detector, and thus the algorithm can be used in a spectral CT reconstruction. Our algorithm uses the known energy-spreading characteristics of Compton scatter ${ }^{1}$ to calculate which energy bin to attribute scatter to.

The algorithm calculates single Compton scatter quantities, neglecting multiple and Rayleigh scatter. We argue that this simplification is justified because most of the scatter affecting micro-CT systems is single scatter, ${ }^{23}$ and omitting the complexity of multiple scatter makes implementation of the algorithm feasible. The algorithm could be extended to include Rayleigh scatter but Compton scatter was our focus due to its energy-shifting characteristic. 


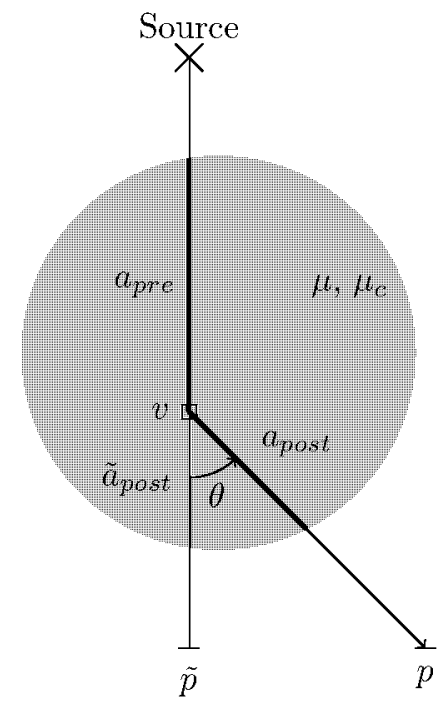

Figure 1: Illustration of some of the quantities used in this derivation. The diagram shows a photon from the source being scattered at $v$ by angle $\theta$, to be counted at detector pixel $p$.

We now present a derivation of the model used by the algorithm. Section 2.1 contains a derivation of the model for the total scatter intensity, then section 2.2 extends the model to include energy-shifting effects. Several of the quantities described are illustrated in Figure 1.

\subsection{Total intensity model}

Let $v$ refer to an arbitrary voxel within the volume being imaged, and let $\tilde{p}$ be the detector pixel such that a line between $\tilde{p}$ and the source passes through $v$. Let $I_{0}$ be the incident open-beam flux at the detector, $A_{\tilde{p}}$ be the area of $\tilde{p}, I_{v}$ be the incident flux on $v$, and $A_{v}$ be the cross-sectional area of $v$. With no material between the source and $v, I_{v}$ can be expressed as

$$
I_{v}=\frac{A_{v}}{A_{\tilde{p}}} I_{0}(\tilde{p}) .
$$

Now let $a_{\text {pre }}$ be the attenuation of the x-ray beam between the source and $v$, such that $0 \leq a_{\text {pre }} \leq 1$ and $a_{p r e}$ is technically a gain type quantity. Then

$$
I_{v}=a_{p r e} \frac{A_{v}}{A_{\tilde{p}}} I_{0}(\tilde{p})
$$

with

$$
a_{p r e}=\exp \left\{-\int_{0}^{x_{v}} \mu(x, \alpha) \mathrm{d} x\right\}
$$

where $x$ represents displacement from the source in the direction of $v, x_{v}$ is the location of $v, \mu(x, \alpha)$ is the total linear attenuation coefficient of the material at $x$, and $\alpha$ is the incident photon energy normalised by the electron rest energy $(511 \mathrm{keV})$.

Let $\mu_{c}$ be the linear attenuation coefficient of Compton scattering. Then the proportion of incident flux that is scattered due to the Compton process is

$$
1-\exp \left\{-\mu_{c}(v, \alpha) \Delta x(v)\right\},
$$

where $\Delta x$ is the path length through $v$ and we assume a constant $\mu_{c}$ within $v$. This expression follows directly from the Beer-Lambert Law. ${ }^{1,24}$ 
Thus the total scattered flux leaving $v$ is given by

$$
a_{p r e} \frac{A_{v}}{A_{\tilde{p}}} I_{0}(\tilde{p})\left(1-\exp \left\{-\mu_{c}(v, \alpha) \Delta x(v)\right\}\right) .
$$

The next step is to evaluate the distribution of this scatter. For this we use the Compton differential crosssection, $\frac{d \sigma_{c}}{d \Omega}$, which describes the probability that an incident photon will be deflected into the elemental solid angle $d \Omega$ when passing through an attenuator containing one scattering centre per unit area. ${ }^{25}$ Integrating this value over the solid angle of a detector pixel as seen from $v$ gives the probability that a photon will be scattered toward that pixel.

From Barrett and Swindell, ${ }^{25}$

$$
\frac{\mathrm{d} \sigma_{c}}{\mathrm{~d} \Omega}(\theta, \alpha)=\frac{r_{0}^{2}}{2}\left[1+\frac{\alpha^{2}(1-\cos \theta)^{2}}{\left(1+\cos ^{2} \theta\right)[1+\alpha(1-\cos \theta)]}\right] \frac{1+\cos ^{2} \theta}{[1+\alpha(1-\cos \theta)]^{2}},
$$

where $\theta$ is the angle of deflection of the photon and $r_{0}$ is the classical electron radius, ${ }^{26} r_{0}=2.818 \times 10^{-13} \mathrm{~cm}$.

To obtain a proportional spatial scatter distribution, we normalise the value of (6) by the complete cross section $\sigma_{c}$. This can be found by

$$
\sigma_{c}=\sigma_{0} f_{K N}(\alpha),
$$

where

$$
f_{K N}(\alpha)=\frac{3}{4}\left[\frac{2(1+\alpha)^{2}}{\alpha^{2}(1+2 \alpha)}+\frac{\ln (1+2 \alpha)}{\alpha}\left(\frac{1}{2}-\frac{1+\alpha}{\alpha^{2}}\right)-\frac{1+3 \alpha}{(1+2 \alpha)^{2}}\right]
$$

is the Klein-Nishina function, ${ }^{27}$ and the Thompson scattering cross-section $\sigma_{0}$ is given by $\sigma_{0}=8 \pi r_{0}^{2} / 3$. Thus the proportion of scattered radiation from $v$ that is directed toward a particular detector pixel $p$ is given by

$$
P(v, p)=\frac{\int_{\Omega_{p, v}} \frac{\mathrm{d} \sigma_{c}}{\mathrm{~d} \Omega}(\theta, \alpha) \mathrm{d} \Omega}{\sigma_{0} f_{K N}(\alpha)}
$$

where $\Omega_{p, v}$ is the solid angle of the pixel $p$ as observed from voxel $v$ and $\theta$ is the deflection angle from $v$ to $p$.

The scattered radiation can also be attenuated between $v$ and $p$. Let $a_{p o s t}$ be this attenuation, defined in a similar manner to $a_{p r e}$ :

$$
a_{\text {post }}=\exp \left\{-\int_{y_{v}}^{y_{p}} \mu\left(y, \alpha^{\prime}\right) \mathrm{d} y\right\}
$$

where $y$ represents position along the line connecting $v$ and $p$, and $\alpha^{\prime}$ is the energy of the scattered photon (see section 2.2). Then the intensity of radiation scattered from $v$ and detected at $p$ is given by

$$
I_{s}(p, v)=\left(a_{p r e} \frac{A_{v}}{A_{\tilde{p}}} I_{0}\right) \cdot\left[1-\exp \left\{-\mu_{c}(v, \alpha) \Delta x(v)\right\}\right] \cdot\left(\frac{\int_{\Omega_{p, v}} \frac{\mathrm{d} \sigma_{c}}{\mathrm{~d} \Omega}(\theta, \alpha) \mathrm{d} \Omega}{\sigma_{0} f_{K N}(\alpha)}\right) \cdot a_{p o s t} .
$$

To enable derivation of a feasible implementation, we now follow the method of Rinkel et al. ${ }^{10}$ in assuming the attenuation factor $a_{\text {post }}$ is equal to that of photons scattered at $\theta=0$, i.e.,

$$
a_{\text {post }} \approx \tilde{a}_{\text {post }}=\exp \left\{-\int_{x_{v}}^{x_{\tilde{p}}} \mu(x, \alpha) \mathrm{d} x\right\},
$$

where $x$ represents position along the line connecting the source and $\tilde{p}$, and $x_{\tilde{p}}$ is the location of $\tilde{p}$ (the detector pixel 'behind' $v$ ). This assumption enables the simplification

$$
a_{\text {pre }} a_{\text {post }}=\exp \left\{-\int_{0}^{x_{p}} \mu(x, \alpha) \mathrm{d} x\right\}=\frac{I(\tilde{p})}{I_{0}(\tilde{p})},
$$

where $I$ is the primary (scatter-free) x-ray intensity and the second equality arises from the Beer-Lambert Law. 
Since the Compton differential cross-section is approximately constant across a pixel, the integral over $\Omega_{p, v}$ can be simplified to

$$
\int_{\Omega_{p, v}} \frac{\mathrm{d} \sigma_{c}}{\mathrm{~d} \Omega}(\theta, \alpha) \mathrm{d} \Omega \approx \frac{\mathrm{d} \sigma_{c}}{\mathrm{~d} \Omega}\left(\theta_{p}, \alpha\right) \cdot \Omega_{p, v}
$$

where $\theta_{p}$ is the angle of deflection from the voxel to the centre of pixel $p$.

Using (13) and (14) in (11) yields

$$
I_{s}(p, v)=\frac{I(\tilde{p})}{I_{0}(\tilde{p})} \frac{A_{v}}{A_{\tilde{p}}} I_{0}(\tilde{p})\left[1-\exp \left\{-\mu_{c}(v, \alpha) \Delta x(v)\right\}\right] \frac{\frac{\mathrm{d} \sigma_{c}}{\mathrm{~d} \Omega}\left(\theta_{p}, \alpha\right) \cdot \Omega_{p, v}}{\sigma_{0} f_{K N}(\alpha)}
$$

for the contribution of scatter from one voxel to one pixel. So the complete scatter field at any pixel $p$ for an incident beam energy $\alpha$ can be calculated by

$$
\begin{aligned}
I_{s}(p) & =\sum_{v} I_{s}(p, v) \\
& =\sum_{v} I(\tilde{p}) \frac{A_{v}}{A_{\tilde{p}}}\left(1-\exp \left\{-\mu_{c}(v, \alpha) \Delta x\right\}\right) \frac{\frac{\mathrm{d} \sigma_{c}}{d \Omega}\left(\theta_{p}, \alpha\right) \cdot \Omega_{p, v}}{\sigma_{0} f_{K N}(\alpha)} .
\end{aligned}
$$

\subsection{Energy change}

As well as being deflected, Compton scattered photons lose energy. This energy loss means that in addition to being detected by a different pixel from the one in the original path, scattered photons may be detected in a different energy bin by an energy-resolving detector. The relationship between original and scattered photon energy is ${ }^{25}$

$$
\alpha^{\prime}=\frac{1}{1 / \alpha+(1-\cos \theta)},
$$

where $\alpha$ is the photon's original energy and $\alpha^{\prime}$ is its energy after scattering through angle $\theta$. This equation rearranges to

$$
\alpha\left(\alpha^{\prime}, \theta\right)=\frac{1}{1 / \alpha^{\prime}-(1-\cos \theta)},
$$

which can be used in (17) to fully model the energy-shifting effect of Compton scattering:

$$
I_{s}\left(p, \alpha^{\prime}\right)=\sum_{v} I\left(\tilde{p}, \alpha\left(\alpha^{\prime}, \theta\right)\right) \frac{A_{v}}{A_{\tilde{p}}}\left(1-\exp \left\{-\mu_{c}\left(v, \alpha\left(\alpha^{\prime}, \theta\right)\right) \cdot \Delta x\right\}\right) \frac{\frac{\mathrm{d} \sigma_{c}}{\mathrm{~d} \Omega}\left(\theta_{p}, \alpha\left(\alpha^{\prime}, \theta\right)\right) \cdot \Omega_{p, v}}{\sigma_{0} f_{K N}\left(\alpha\left(\alpha^{\prime}, \theta\right)\right)} .
$$

We have implemented the model in (20). The current implementation is in Python (using NumPy), but could be accelerated considerably by converting it to a language such as $\mathrm{C}$ or $\mathrm{C}++$ and applying a number of optimisations. We have not done this as the principal aim of this project was to evaluate the effectiveness and utility of the algorithm, not a particular implementation of it.

Our implementation currently estimates the amount of scatter generated within a single slice of an object. The mathematics given here are not limited to this case, and the program can be extended to operate on conebeam data where scatter is a much greater problem. ${ }^{23}$ For the purposes of this paper, the single-slice operation has been retained as it makes results simpler to interpret and reproduce, and reduces the time required to run Monte Carlo simulations for validation. 


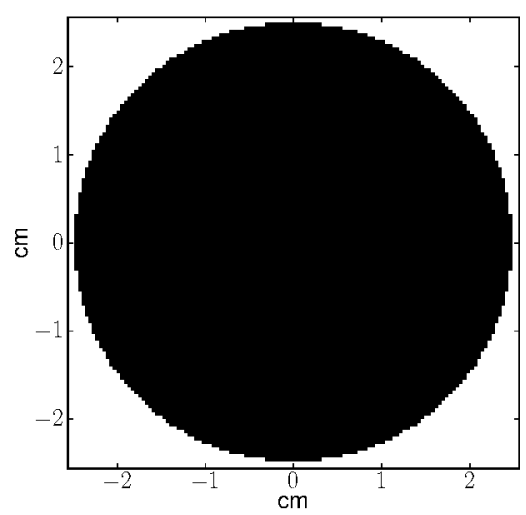

(a)

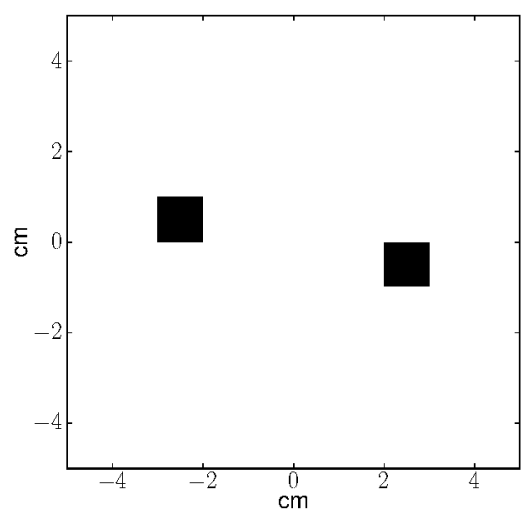

(b)

Figure 2: The two phantoms used for testing. (a) is a PMMA cylinder, (b) is two blocks of cortical bone.

\section{RESULTS}

We compared the output of the algorithm to the scatter predicted by the Monte Carlo simulator BEAMnrc. ${ }^{28}$ In the configuration of BEAMnrc we disabled Rayleigh scatter and Compton binding effects so that we could directly compare the results. Variance reduction techniques were also disabled. We used two simulated phantoms for the testing; a cross section of each is shown in Figure 2. The algorithm was tested with a parallel beam source illuminating the phantoms from above.

The first phantom, shown in Figure 2(a), was a $2.5 \mathrm{~cm}$ radius PMMA cylinder. The gap between the bottom of the cylinder and the detector was $1.5 \mathrm{~cm}$. This phantom was to test the operation on a simple homogeneous structure. The second phantom, shown in Figure 2(b), was made of two blocks of cortical bone, each with a $1 \mathrm{~cm}$ square profile, vertically offset by $1 \mathrm{~cm}$ and with a $4 \mathrm{~cm}$ gap between them horizontally. The detector was located $4 \mathrm{~cm}$ below the bottom edge of the lower block. This phantom was intended to test the operation on an object with inhomogeneities. Different materials were used for the two phantoms to test the operation of the algorithm on materials with different Compton cross-sections.

We performed the testing with each of two monochromatic x-ray sources, $50 \mathrm{keV}$ and $30 \mathrm{keV}$, and energy bins of $4 \mathrm{keV}$ width, with the source energy in the middle of the highest bin. We used a monochromatic source so that the results would be simpler to interpret, and we chose 30 and $50 \mathrm{keV}$ as they fall within the diagnostic energy range. We used a $4 \mathrm{keV}$ bin width because this is around the greatest resolution achievable by current detectors. The Compton cross-sections and complete attenuation coefficients used by our algorithm were obtained from the database distributed with BEAMnrc which in turn originates from the online XCOM program; ${ }^{29}$ the values are given in Table 1.

Figure 3 shows the intensities predicted for the PMMA cylinder. All scatter intensities shown in this and subsequent figures have been normalised by the total flat-field (open beam) intensity to facilitate comparison. For the $48-52 \mathrm{keV}$ energy bin the form of the profile produced by our algorithm closely matches that of the Monte Carlo simulation, and the magnitude matches well for most of the detector width. In the lower energy bin, $44-48 \mathrm{keV}$, the output of our algorithm matches that of BEAMnrc reasonably well, apart from in the central region of the detector, where our algorithm estimates zero scatter but BEAMnrc does not. Inspection of the scales on the $\mathrm{y}$-axes shows that the vast majority of scattered radiation remains in the $48-52 \mathrm{keV}$ bin. Plots are not shown for bins lower than $44 \mathrm{keV}$ as the intensity levels are negligible.

Table 1: Total $(\mu)$ and Compton $\left(\mu_{c}\right)$ linear attenuation coefficients of phantom materials. ${ }^{29}$ Units: $\mathrm{cm}^{-1}$.

\begin{tabular}{c|cccc} 
& $\mu_{c}(30 \mathrm{keV})$ & $\mu(30 \mathrm{keV})$ & $\mu_{c}(50 \mathrm{keV})$ & $\mu(50 \mathrm{keV})$ \\
\hline PMMA & 0.321 & 0.334 & 0.206 & 0.236 \\
Cortical bone & 0.356 & 2.423 & 0.334 & 0.761
\end{tabular}




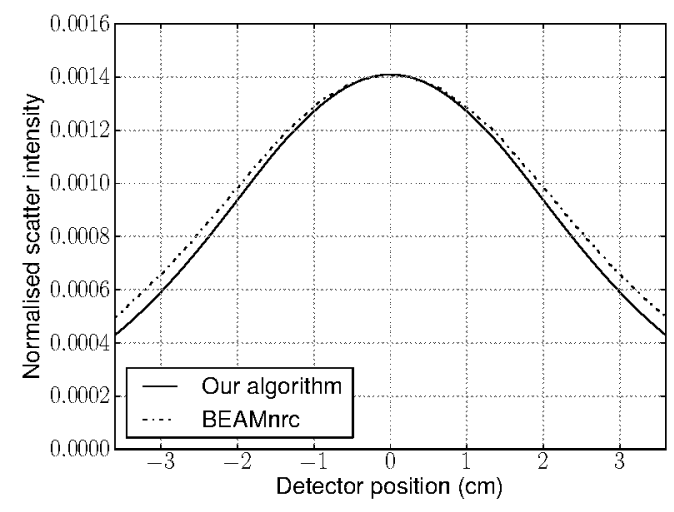

(a)

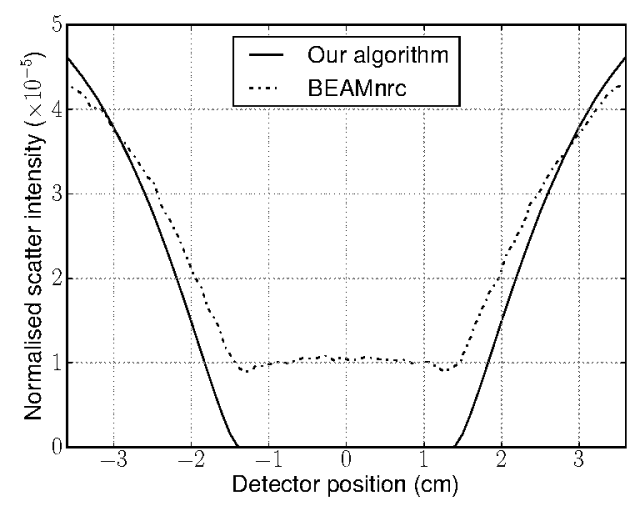

(b)

Figure 3: Normalised scatter intensity with the PMMA cylinder phantom, $50 \mathrm{keV}$ source. (a) is the scatter in the $48-52 \mathrm{keV}$ bin, (b) is in the $44-48 \mathrm{keV}$ bin.

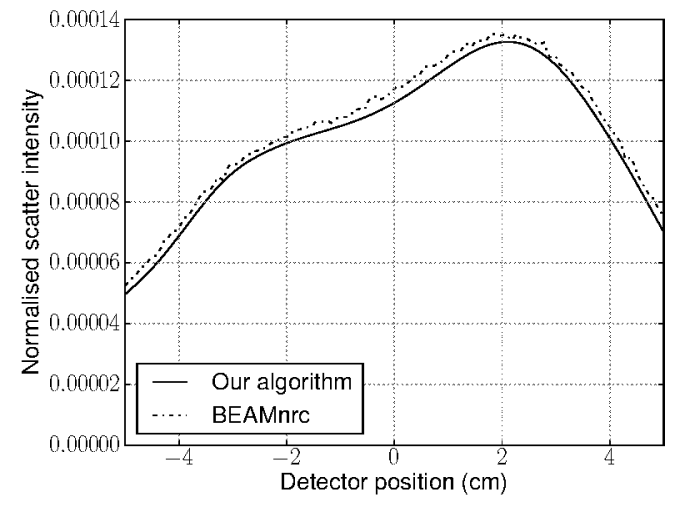

(a)

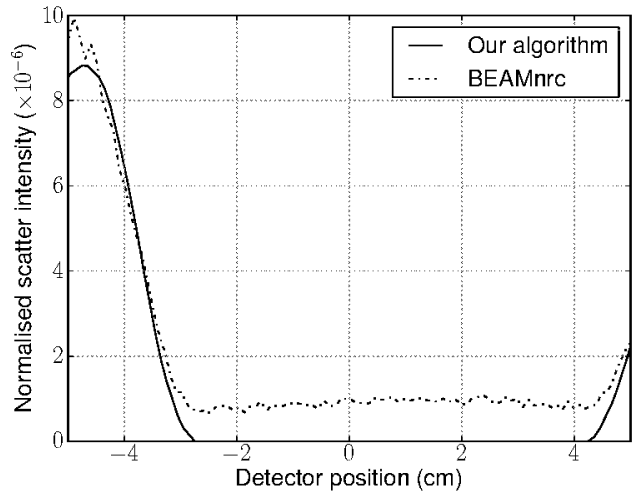

(b)

Figure 4: Normalised scatter intensity with the bony phantom, $50 \mathrm{keV}$ source. (a) is the scatter in the $48-52 \mathrm{keV}$ bin, (b) is in the $44-48 \mathrm{keV}$ bin.

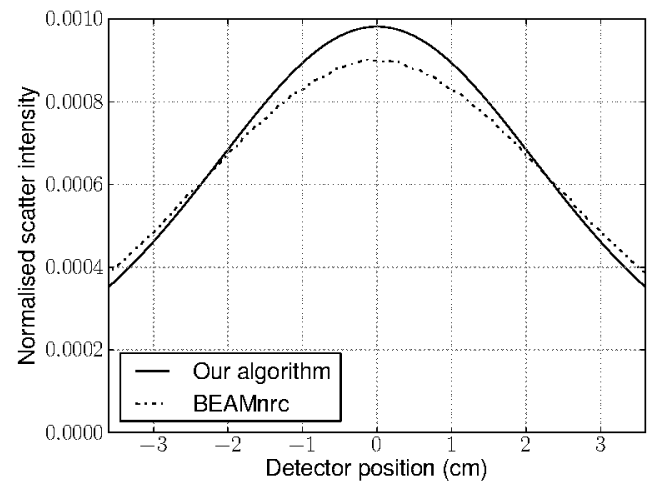

(a)

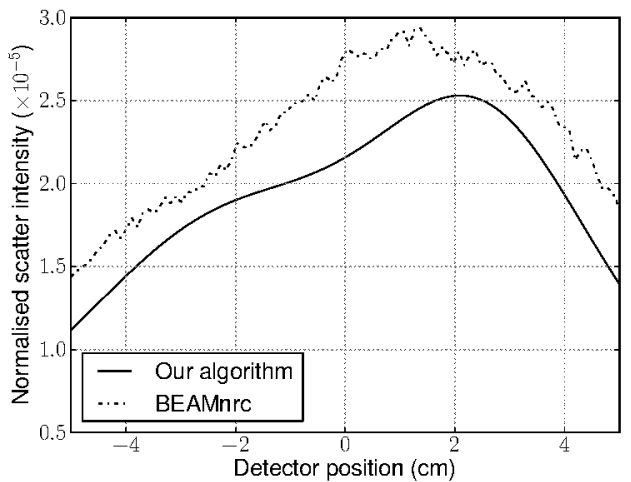

(b)

Figure 5: Normalised scatter intensity with a $30 \mathrm{keV}$ source, in the $28-32 \mathrm{keV}$ bin. (a) is the scatter from the PMMA cylinder, (b) is from the bones. 
Figure 4 shows the intensities predicted for the bony phantom. In the upper energy bin our algorithm again closely matches the profile of the Monte Carlo output but predicts a slightly smaller magnitude. The estimate for the lower energy bin again matches BEAMnrc reasonably well toward the edges of the detector but falls to zero in the centre while BEAMnrc does not. The overall scatter intensity from this phantom is smaller than that of the PMMA cylinder, as could be expected from the much smaller volume of material.

To confirm that our algorithm works across the diagnostic energy range, we repeated the tests with a $30 \mathrm{keV}$ source. Figure 5(a) shows that our algorithm still agrees with the amount produced by BEAMnrc although the form of the scatter does not match quite as closely as it does at $50 \mathrm{keV}$. Conversely, Figure 5 (b) shows that for the bony phantom the estimate has a good shape but is smaller in magnitude. In fact we were able to reproduce the profiles generated by BEAMnrc very closely by running our algorithm with slightly different coefficients, however we have not shown these result as this experiment was intended to evaluate the operation of the algorithm without any empirical calibration.

Our algorithm was much faster to run than a BEAMnrc simulation. Calculating the scatter intensity for a single view angle of the cylinder phantom with our new algorithm took 3.8 seconds on a single processor, while the BEAMnrc simulation took a little over 4 hours using 8 processors.

\section{DISCUSSION}

The results in section 3 show that our new algorithm performs well. Apart from missing some scatter near the centre of the detector for energy bins below the source energy, both the form and magnitude agree either reasonably or very well with the output of BEAMnrc. The scatter in the $44-48 \mathrm{keV}$ bin is made up of photons that have lost enough energy to 'bin-hop'. We conjecture that the intensity predicted by BEAMnrc through the central detector region for this bin comprises photons which have been multiply scattered; such photons are not modelled by our algorithm. The basis for this claim is that for a photon to lose enough energy to bin-hop, it must either scatter at a large angle or experience multiple scatter events. Since scatter reaching the centre of the detector can only have been deflected by a small angle, it must have been scattered more than once to appear in a lower energy bin.

The results presented here suggest that the amount of scatter that hops between energy bins of a practical size is not significant enough to warrant inclusion in an estimation scheme. Accurate estimation of intra-bin scatter is much more important for effective scatter compensation, and our algorithm performs well at this task. Removal of the logic that accounts for inter-bin scatter would simplify and accelerate the software slightly.

While estimating inter-bin scatter is of low importance, this should not be confused with estimating the amount of scatter produced at different energies within a polychromatic (broad-band) spectrum. Comparison of the scatter quantities at the two energies tested in this paper shows that different amounts of scatter are produced at each energy. This leads us to the conclusion that scatter estimation and compensation on a bin-by-bin basis is worthwhile for spectral CT. Our algorithm has the ability to do this and can work with the spectral variation of the Compton scattering cross-section. This type of spectrally-aware compensation can be applied independently to each bin if inter-bin scatter is neglected.

Our algorithm requires knowledge of both $\mu$ and $\mu_{c}$ to operate. The total attenuation can be obtained from an initial reconstruction, as is the case with other reconstruction-based iterative algorithms. Our suggested method of obtaining $\mu_{c}$ is simply by scaling the value for $\mu$, based on a simple segmentation procedure. This involves using thresholding to distinguish between air, soft tissue, and bone, then multiplying $\mu$ by a predetermined scaling factor according to the type of tissue comprising each voxel. This works because soft tissues all have a similar ratio of $\mu_{c}$ to $\mu$ (about $79 \%$ at $50 \mathrm{keV}$ ), and bone and air similarly have known ratios. It is possible to tailor the particular scaling factors according to the type of scan being performed, since the types of tissue likely to be encountered are usually known. For example, when performing a chest CT it is usually safe to assume the presense of lung tissue and ribs, so the soft-tissue ratio can be biased towards that of inflated lung while the bone ratio can be set to that of rib tissue.

Several approaches can be taken to the use of contrast agents, e.g., iodine or gadolinium. With intravenous contrast, the volume of contrast will be small compared to the rest of the body, so it should be acceptable to treat contrast voxels as bone (since they will be segmented as such due to their higher attenuation). In the case of 
orally administered contrast, the bolus may be present in a larger volume. This could be addressed by choosing the scaling factor for 'bone' to be closer to that of the contrast. It may also be feasible to include contrast as a separate segmentation target.

\section{CONCLUSIONS AND FUTURE WORK}

We have derived an algorithm for the estimation of Compton scatter based on a reconstruction of a micro-CT scan. We have implemented this algorithm and verified its results against the output of the BEAMnrc Monte Carlo simulator. We found that our program is effective at estimating single scatter. For micro-CT single scatter is the dominant process, although multiple scatter is more prominent in energy bins below the source energy. We also found that very little scatter crosses bin boundaries, so this phenomenon can be omitted from the model.

The amount of scatter varies with the energy of the incident radiation, so energy-resolved CT reconstruction will benefit from the use of a scatter compensation technique that accounts for this behaviour. Our algorithm is suitable for this task, and is much faster than running a Monte Carlo simulation.

There are three main improvements needed for our algorithm. First, the program needs to account for binding effects in Compton scatter, so that the actual amount of Compton scatter can be correctly estimated. Next, Rayleigh scatter should be included in the model. This would require the use of another scaling factor to obtain $\mu_{r}$ based on the reconstructed $\mu$. Finally, the geometry assumed by the program should be converted to cone-beam. The first detector style to be implemented would be flat-panel as that is the style used in our MARS scanner, but consideration will also be given to the curved detector styles.

\section{REFERENCES}

[1] Johns, H. E. and Cunningham, J. R., [The Physics of Radiology], Charles C Thomas, Springfield, Illinois, USA, 4th ed. (1983).

[2] Strutt, J. W., "On the transmission of light through an atmosphere containing small particles in suspension, and on the origin of the blue of the sky," Philos. Mag. 47, 375-384 (1899).

[3] Compton, A. H., "A quantum theory of the scattering of x-rays by light elements," Physical Review 21(5), 483-502 (1923).

[4] Joseph, P. and Spital, R., "The effects of scatter in x-ray computed tomography," Med. Phys. 9(4), 464-472 (1982).

[5] Hunter, A. K. and McDavid, W. D., "Characterization and correction of cupping effect artefacts in cone beam CT," Dentomaxillofacial Radiology 41, 217-223 (2012).

[6] Bucky, G., "Über die Ausschaltung der im Objekt entstehenden Sekundärstrahlen bei Röntgenaufnahmen," Verh. Deutsch Röntgengesell 9, 30-32 (1913).

[7] Neitzel, U., "Grids or air gaps for scatter reduction in digital radiography: A model calculation," Med. Phys. 19(2), 475-481 (1992).

[8] Colijn, A. and Beekman, F., "Accelerated simulation of cone beam x-ray scatter projections," IEEE Trans. Med. Imag. 23(5), 584-590 (2004).

[9] Wagner, F., Macovski, A., and Nishimura, D., "Two interpolating filters for scatter estimation," Med. Phys. 16(5), 747-757 (1989).

[10] Rinkel, J., Gerfault, L., Estève, F., and Dinten, J.-M., "A new method for x-ray scatter correction: first assessment on a cone-beam CT experimental setup," Phys. Med. Biol. 52, 4633-4652 (2007).

[11] Seibert, J. and Boone, J., "X-ray scatter removal by deconvolution," Med. Phys. 15(4), 567-575 (1988).

[12] Ohnesorge, B., Flohr, T., and Klingenbeck-Regn, K., "Efficient object scatter correction algorithm for third and fourth generation CT scanners," Eur. Radiol. 9(3), 563-569 (1999).

[13] Rührnschopf, E.-P. and Klingenbeck, K., "A general framework and review of scatter correction methods in x-ray cone-beam computerized tomography. Part 1: Scatter compensation approaches," Med. Phys. 38(7), $4296-4311$ (2011).

[14] Taguchi, K., Zhang, M., Frey, E. C., Xu, J., Segars, W. P., and Tsui, B. M. W., "Image-domain material decomposition using photon-counting CT," in [Proceedings of SPIE], 6510, 651008 (2007). 
[15] Tapiovaara, M. and Wagner, R. F., "SNR and DQE analysis of broad spectrum x-ray imaging," Phys. Med. Biol. 30(6), 519-529 (1985).

[16] Ballabriga, R., Campbell, M., Heijne, E. H. M., Llopart, X., and Tlustos, L., "The Medipix3 prototype, a pixel readout chip working in single photon counting mode with improved spectrometric performance," IEEE Trans. Nucl. Sci. 54(5), 1824-1829 (2007).

[17] Walsh, M. F., Opie, A. M. T., Ronaldson, J. P., Doesburg, R. M. N., Nik, S. J., Mohr, J. L., Ballabriga, R., Butler, A. P. H., and Butler, P. H., "First CT using Medipix3 and the MARS-CT-3 spectral scanner," J. Inst. 6, C01095 (2011).

[18] Giersch, J., Niederlöhner, D., and Anton, G., "The influence of energy weighting on x-ray imaging quality," Nucl. Instrum. Meth. A 531(1), 68-74 (2004).

[19] Niederlohner, D., Karg, J., Giersch, J., Firsching, M., and Anton, G., "Practical aspects of energy weighting in x-ray imaging," in [IEEE Nucl. Sci. Symp. Conf. Rec.], 5, 3191-3194, IEEE (2004).

[20] Zainon, R., Ronaldson, J., Janmale, T., Scott, N., Buckenham, T., Butler, A., Butler, P., Doesburg, R., Gieseg, S., Roake, J., et al., "Spectral CT of carotid atherosclerotic plaque: comparison with histology," Eur. Radiol. , 1-8 (2012).

[21] Schlomka, J. P., Roessl, E., Dorscheid, R., Dill, S., Martens, G., Istel, T., Bäumer, C., Herrmann, C., Steadman, R., Zeitler, G., Livne, A., and Proska, R., "Experimental feasibility of multi-energy photoncounting K-edge imaging in pre-clinical computed tomography," Phys. Med. Biol. 53, 4031-4047 (Jul. 2008).

[22] Bones, P. J., Butler, A. P. H., Ronaldson, J. P., and Opie, A. M. T., "Development of a CT scanner based on the Medipix family of detectors," in [Developments in X-Ray Tomography VII, Proc. SPIE], 7804 (Aug. 2010).

[23] Kyriakou, Y. and Kalender, W., "X-ray scatter data for flat-panel detector CT," Physica Medica 23(1), 3-15 (2007).

[24] Beer, A., "Bestimmung der Absorption des rothen Lichts in farbigen Flüssigkeiten," Ann. Phys. Chem 86(2), 78-90 (1852).

[25] Barrett, H. H. and Swindell, W., [Radiological Imaging: The Theory of Image Formation, Detection, and Processing], Academic Press (1981).

[26] Mohr, P. J., Taylor, B. N., and Newell, D. B., "CODATA recommended values of the fundamental physical constants: 2006," J. Phys. Chem. Ref. Data. 37(3), 1187-1284 (2008).

[27] Klein, O. and Nishina, Y., "The scattering of light by free electrons according to Dirac's new relativistic dynamics," Nature 122(3072), 398-399 (1928).

[28] Rogers, D. W. O., Walters, B., and Kawrakow, I., "BEAMnrc users manual," Tech. Rep. 509(A), National Research Council of Canada (2006).

[29] Berger, M. J., Hubbell, J. H., Seltzer, S. M., Chang, J., Coursey, J. S., Sukumar, R., Zucker, D. S., and Olsen, K., "XCOM: Photon cross sections database (version 1.5)," Tech. Rep. 87-3597, National Institute of Standards and Technology, Gaithersburg, MD (2010). Available at http://physics.nist.gov/xcom. 